APJ IN PRESS

Preprint typeset using $\mathrm{LATEX}_{\mathrm{E}}$ style emulateapj v. 2/19/04

\title{
THE UNUSUAL X-RAY MORPHOLOGY OF NGC 4636 REVEALED BY DEEP CHANDRA OBSERVATIONS: CAVITIES AND SHOCKS CREATED BY PAST AGN OUTBURSTS
}

\author{
A. Baldi, W. Forman, C. Jones, R. Kraft, P. Nulsen
}

Harvard-Smithsonian Center for Astrophysics

\author{
E. Churazov \\ Max-Planck-Institute für Astrophysik \\ L. DAvid, S.Giacintucci \\ Harvard-Smithsonian Center for Astrophysics \\ ApJ in press
}

\begin{abstract}
We present Chandra ACIS-I and ACIS-S observations $(\sim 200 \mathrm{ks}$ in total $)$ of the X-ray luminous elliptical galaxy NGC 4636, located in the outskirts of the Virgo cluster. A soft band $(0.5-2 \mathrm{keV})$ image shows the presence of a bright core in the center surrounded by an extended X-ray corona and two pronounced quasi-symmetric, $8 \mathrm{kpc}$ long, arm-like features. Each of this features defines the rim of an ellipsoidal bubble. An additional bubble-like feature, whose northern rim is located $\sim 2 \mathrm{kpc}$ south of the north-eastern arm, is detected as well. We present surface brightness and temperature profiles across the rims of the bubbles, showing that their edges are sharp and characterized by temperature jumps of about 20-25\%. Through a comparison of the observed profiles with theoretical shock models, we demonstrate that a scenario where the bubbles were produced by shocks, probably driven by energy deposited off-center by jets, is the most viable explanation to the X-ray morphology observed in the central part of NGC 4636. As a confirmation to this scenario, radio jets extending towards the bubbles and a central weak X-ray and radio source are detected and are most likely the signs of AGN activity which was more intense in the past. A bright dense core of $\sim 1 \mathrm{kpc}$ radius is observed at the center of NGC 4636. A sharp decline in surface brightness from the core to the ambient gas is observed and is not accompanied by a variation in the temperature and thus could not be in thermal pressure equilibrium. However the bright core could be a long lived feature if the radio jets are acting as a balancing factor to thermal pressure or if the bright core is produced by steep abundance gradients.

Subject headings: galaxies: individual (NGC 4636) - galaxies: ISM - galaxies: nuclei - X-rays: galaxies
\end{abstract}

\section{INTRODUCTION}

It is well known that active galactic nuclei (AGN) play an important role in the evolution of the hot gas in both individual galaxies and clusters of galaxies (e.g. Churazov et al. 2000; Brüggen \& Kaiser 2002; Cavaliere et al. 2002; Hoeft \& Brüggen 2004; Forman et al. 2005; (McNamara \& Nulsen 2007). The socalled 'AGN feedback' can cause re-heating of the central cooling regions of a cluster and balance the cooling due to the X-ray emission (see McNamara \& Nulsen 2007, for a review). AGN are also effective in shaping the morphology of the hot gas halos around individual galaxies and clusters, e.g. giving rise to cavities and bubbles (Bîrzan et al. 2004).

While several examples of subsonic bubble inflation due to AGN outbursts in individual galaxies are present in the literature (e.g. NGC 507, M 84; Kraft et al. 2004; Finoguenov et al. 2008), supersonic bubble expansion, giving rise to shocks in the hot X-ray emitting gas, has only been observed in a handful of cases (e.g. M 87, NGC 4552; Forman et al. 2005; Machacek et al. 2006). Another possible case of supersonic bubble expansion could be that of NGC 4636, the dominant galaxy of a group on the outskirts of the Virgo Cluster $\left(10^{\circ}\right.$ or $2.6 \mathrm{Mpc}$ on the sky to the south of M87, at a distance to NGC 4636 of $15 \mathrm{Mpc}$; Tonry et al. 2001). This galaxy has been observed extensively by every major X-ray imaging observatory to date. Spectral imaging with Einstein showed the galaxy to be surrounded by an extensive halo of hot gas with a temperature of $0.78 \mathrm{keV}$ (Forman et al. 1985). Further studies using ROSAT and ASCA found gradients in both the temperature and abundance profiles, along with a very extended halo (Awaki et al. 1994; Trinchieri et al. 1994; Matsushita et al. 1997; Finoguenov \& Jones 2000; Buote 2000; O'Sullivan et al. 2003). The metallicity is about solar in the central $9 \mathrm{kpc}\left(2^{\prime}\right)$ region, decreasing to $0.2-0.3$ solar at $\sim 45 \mathrm{kpc}\left(10^{\prime}\right)$ from the galaxy center (Matsushita et al. 1997). More recently, NGC 4636 has been observed by both Chandra and XMM-Newton. The initial Chandra ACIS-S observation showed an unusual morphology in the core of the galaxy, most notably quasi-symmetric spiral arm-like features and a possible ring of high surface brightness emission, thought to be the product of shocks driven by a previous AGN outburst (Jones et al. 2002). As a confirmation to the outburst driven shock scenario, a change of the gas temperature across one of the arms was observed as well. From the same Chandra data, Ohto et al. (2003) performed a detailed spectral analysis, suggesting the presence of a cavity in the X-ray halo on the west side of the core, with AGN activity being the most likely cause 
also in this case. In this scenario, plasma from the AGN jets may be able to displace the gas of the X-ray halo, leaving an apparent void as currently observed in the X-ray morphology. Using data from XMM-Newton and Chandra, O'Sullivan et al. (2005) mapped the temperature and abundance structure of the inner halo of NGC 4636 , confirming the detection of a cavity to the west of the galaxy and revealing the presence of a plume of cool metal-rich gas extending beyond the cavity to the southwest. Both cavity and plume appear to be the product of past AGN activity. Moreover, their abundance determination shows a depression in the central few $\mathrm{kpc}(\sim 0.2$ solar $)$ in disagreement with the results of Matsushita et al. (1997).

In this paper we take advantage of the long Chandra ACIS-I observations $(\sim 150 \mathrm{ks})$ combined with the previously observed ACIS-S exposure ( $\sim 40 \mathrm{ks})$ to analyze in more detail the features revealed by the previous Chandra and XMM-Newton observations. In particular we are able to study in more detail the morphology and temperature structure in the observed cavities and look for gradients in temperature and abundance to test the shock scenario invoked for their formation (Jones et al. 2002; Ohto et al. 2003; O'Sullivan et al. 2005). Moreover we will investigate with unprecedented detail the morphology and the properties of the hot gas in the galaxy core.

At the distance of NGC 4636 (15 Mpc; Tonry et al. 2001), $1^{\prime \prime} \approx 70 \mathrm{pc}$. Throughout the paper, all the uncertainties are quoted at $1 \sigma(68 \%)$ for one interesting parameter, unless otherwise stated. The abundance estimates are relative to the cosmic values given in Anders \& Grevesse (1989).

\section{CHANDRA DATA PREPARATION AND ANALYSIS}

NGC 4636 was observed twice by ACIS-S and twice by ACIS-I. In our analysis, we use the longer ACIS-S observation (performed on 2000, January 26; ObsID: 323 ) and both the ACIS-I observations, performed one immediately after the other on 2003, February 14 (ObsID: 3926) and on 2003, February 15 (ObsiD: 4415). The Chandra data analysis has been performed using CIAO v3.4, which applies the newest ACIS gain maps, time-dependent ACIS gain corrections, and ACIS charge transfer inefficiency corrections. The background light curve during the observation has been examined to detect periods of high background following Markevitch et al. (2003). A flaring episod (lasting $\sim 15 \mathrm{ks})$ is observed during the ACIS-S observation, reducing the usable exposure time to $\sim 37 \mathrm{ks}$. No significant flaring episodes are detected during the two ACIS-I observations, giving a total good integration time for the ACIS-I detector of $\sim 149$ ks.

\subsection{Background Subtraction}

We use the "blank-field" observations, processed identically to the galaxy observations (i.e. as described above) and reprojected onto the sky using the aspect information from the galaxy pointings. For the ACIS-I observations, the "blank-fields" are renormalized to the background of the observation using the ACIS-S2 chip. For the ACIS-S observation, the chip used for the renormalization is ACIS-S1 owing to the extended source emission on S3. The energy band from 9.5 to $12 \mathrm{keV}$ (mostly dominated by charged particles) is used to perform the normalization.

We follow the procedure of Vikhlinin et al. (2005), to check if the diffuse soft X-ray background could be an important background component in our observation. A spectrum from the source-free region of the S2 chip was extracted, the renormalized "blank-field" background was subtracted and the background subtracted spectrum was fitted in XSPEC v11.3.2p (in the 0.4-1 keV band) with an unabsorbed mekal model, whose normalization is allowed to be negative. The residuals are found to be negligible and consistent with zero in all three observations, suggesting that no additional soft background correction is necessary for our datasets.

\section{RESULTS}

\subsection{X-ray morphology and surface brightness}

Fig. 17a shows the merged $0.3-2 \mathrm{keV}$ Chandra image of all three observations (two ACIS-I and one ACIS-S observation). The image was "flat-fielded" using an appropriate exposure map weighted for the emission of a $0.6 \mathrm{keV}$ thermal plasma. The galaxy presents a very bright central core of radius $\sim 1 k p c\left(15^{\prime \prime}\right)$ centered on the nucleus. A lower surface brightness region surrounds the nucleus extending out to $\sim 6 \mathrm{kpc}\left(80^{\prime \prime}\right)$. Two quasisymmetric arm-like features are embedded in this lower surface brightness emission. These features were previously reported by Jones et al. (2002) who analyzed the shorter ACIS-S observation. However, combining these data with the two ACIS-I observations we are able to observe that the south-western arm is clearly part of an X-ray cavity extending as far as $\sim 9 \mathrm{kpc}\left(2^{\prime}\right)$ from the center. The hint of a similar structure can be observed in coincidence with the north-eastern arm-like feature. It is not clear from the X-ray image whether there is a cavity in this case. However the cavities are more evident if we remove the contribution from the general diffuse emission of the galaxy. Fig. 1 $\mathrm{b}$ shows the merged Chandra image in the $0.3-2 \mathrm{keV}$ band where a $\beta$-model fitted to the galaxy diffuse emission was subtracted. This processing allowed to highlight fainter structures. Indeed, both the south-western and the north-eastern cavities are clearly visible in this Figure. Moreover two additional quasisymmetric cavities can be seen, just to the East of the nucleus and to the North-West of it, with the rims of the former being better defined than the latter.

The radio data overlaid on the X-ray image (Fig. 2) shows a correlation with the cavities. However, the radio lobes do not fill the SW cavity, or break through the cavity boundaries as in other galaxies presenting cavities (e.g. M 84, Finoguenov et al. 2008). However, the lobes detected at $610 \mathrm{MHz}$ (Giacintucci et al. in prep.) with the Giant Metrewave Radio Telescope (GMRT) extend further than those observed at $1.4 \mathrm{GHz}$ and also the $\mathrm{NE}$ cavity seems to be filled by plasma detected at $235 \mathrm{MHz}$ with the GMRT (Giacintucci et al. in prep.). The radio lobes are weak, having a luminosity at $1.4 \mathrm{GHz}$ of $\sim 1.4 \times 10^{38} \mathrm{erg} \mathrm{s}^{-1}$. However the plasma creating the cavities remains partially undetected by the radio observations of NGC 4636 performed so far.

Figure 3 shows Chandra images of NGC 4636 in two different bands: $0.3-0.9 \mathrm{keV}$ and $0.9-1.3 \mathrm{keV}$. A $2^{\prime \prime}$ width gaussian smoothing was applied to them to enhance the features visible in the two images. The morphology is 
quite different between them, with the X-ray arm-like features becoming prominent only at energies larger then $0.9 \mathrm{keV}$, a possible indication that the temperature in these features is higher than in the surrounding hot interstellar medium of the galaxy.

\subsection{Global X-ray properties}

The surface brightness profile of NGC 4636 is shown in Figure 4. The emission does not show a central peak and is quite flat at the center, then it starts to decline monotonically at $\sim 5^{\prime \prime}(0.35 \mathrm{kpc})$ from the center, showing another 'plateau' at $25^{\prime \prime}<r<60^{\prime \prime}(1.75 \mathrm{kpc}<r<$ $4.2 \mathrm{kpc}$ ). Both these features can be noticed looking at the Chandra image (Fig. 1 1 ). To study the radial spectral properties, we subdivided the emission from the galaxy into 25 annuli centered on the X-ray peak. We extracted a spectrum from each annulus (excluding the point sources) using specextract, which generates source and background spectra building appropriate RMFs and ARFs. The background is taken from the re-normalized blank field observations using the same region as the source. The spectra were analyzed using XSPEC v12 (Arnaud 1996) and fitted with a single-temperature APEC model (Smith et al. 2001), combined with the XSPEC projct deprojection model. The free parameters in the model are the gas temperature $k T$, the gas metallicity $Z$ and the normalization. The spectral band considered is $0.5-4 \mathrm{keV}$. All the spectra were rebinned to have at least 20 counts per bin. We have measured $N_{H}$ from the X-ray data, finding it consistent (within $1 \sigma$ ) with the Galactic value along the line of sight, as derived from radio data $\left(1.9 \times 10^{20} \mathrm{~cm}^{-2}\right.$; Stark et al. 1992). The deprojected temperature profiles of NGC 4636 are shown in Figure $5 \mathrm{~b}$. No abundance profile trend was detected, we therefore decided to fix the abundance at $Z=0.5$ times solar. This is in agreement with the findings of O'Sullivan et al. (2005), who also found no trend in $Z(r)$ for the azimuthally-averaged data. Interestingly, they did however find a strong azimuthal abundance variation. The temperature shows clear evidence for a decline in the center where $k T \sim 0.5 \mathrm{keV}$, and increases toward the outskirts of the galaxy $(k T \gtrsim 0.8 \mathrm{keV})$. Several substructures, due to the complex $\widetilde{X}$-ray morphology of the galaxy, are observed. The electron density profile derived from the deprojection (Fig. [5b) shows instead a strong central peak and a 'plateau' coincident with the one observed in the surface brightness profile located at $2 \mathrm{kpc} \lesssim r \lesssim 4.5 \mathrm{kpc}$ from the center.

\subsection{The X-ray Bubbles}

The complex X-ray morphology of NGC 4636 is mainly constituted by bubbles and cavities, whose properties cannot be determined with a simple radial analysis. Therefore, we studied the features observed in the Xray image in detail, performing a spatially resolved spectral analysis on these regions, looking for evidence of the feedback from the central AGN in the surrounding hot interstellar and intergalactic medium.

\subsubsection{The $S W$ Bubble}

The most prominent features observed in the Chandra image are clearly the two quasi symmetric $8 \mathrm{kpc}$ long Xray arm-like structures, already observed by Jones et al.
(2002) and by O'Sullivan et al. (2005). The SW arm (SW1) is however part of a cavity which extends at least $\sim 5 \mathrm{kpc}$ in radius to the North (Fig. 6), although the northern boundary of the bubble (SW2) is not as sharp and well defined as the southern one. We performed a spectral analysis in a strip perpendicular to the SW arm-like feature, dividing the strip into rectangles $\sim 0.5^{\prime}$ wide (Fig. 7), to look for variations in temperature or metal abundance. Although the metallicity does not vary significantly across the bubble rim, a sharp variation in the temperature was clearly detected coincident with SW1 (Fig. 8) showing a temperature decrease from $k T \sim 0.75 \mathrm{keV}$ to $k T \sim 0.64 \mathrm{keV}$, well above the measurement errors $(\sim 0.01 \mathrm{keV})$. A temperature jump was not observed across SW2 most likely because of the complicated geometry of the X-ray emission. Indeed, SW2 seems to be partially embedded in another bubble-like feature located just North of it.

\subsubsection{The NE and the E Bubble}

The symmetry of the X-ray arm-like features is highly suggestive of the presence of a symmetric bubble NE of the nucleus of NGC 4636. However the southern rim of the bubble is not clearly visible and it looks instead to be embedded in another round shaped bubble located to the East of the nucleus. If we examine the surface brightness profile of the NE cavity we find a shape which is very similar to the SW bubble (Fig. 9a). Performing a spectral analysis across the northern cavity rim NE1 (Fig. 17), we also observe a temperature jump across the rim (Fig. 9b). However, the scenario in this part of the galaxy is more complex because of the presence of an additional feature just East of the nucleus with the shape of another cavity. This cavity looks less elongated than the other two cavities observed. Therefore, we preferred to analyze the temperature variation across its southern rim E1 (the northern boundary E2 could be contaminated by the presence of the NE cavity) using partial annuli instead of rectangular regions (Fig. 77). The surface brightness profile has a shape similar to the one across the NE cavity, while the temperature profile shows a temperature jump similar to the one observed in the other two cavities (Fig. 10).

\subsubsection{The origin of the cavities: a simple shock model}

The most likely scenario for the origin of the cavities observed in the X-ray morphology of NGC 4636 is that they were the result of successive outbursts of the central AGN. In this scenario the jet propagated rapidly from the center, creating a long thin cavity which then inflated in all directions. Perpendicular to the axis of the jet, the expansion has an approximate cylindrical symmetry, motivating our choice to use rectangular regions and therefore assume cylindrical symmetry. The expanding cavities drive shocks into the surrounding gas.

A 1-dimensional, cylindrically symmetric, timedependent hydrodynamic model was used to investigate the properties of these shocks for the SW bubble. In this model the sound speed in the relativistic gas that fills the cavity (the piston that drives the shock) is assumed very high, keeping the pressure in the piston nearly uniform. The ratio of the pre-shock pressure to the post-shock 
pressure determines the strength of the shock. As a result, the shock is weakest (slowest) in the region close to the AGN and fastest in the region farthest from the AGN.

The undisturbed gas was assumed to be isothermal, with electron density $n_{\mathrm{e}, \mathrm{gas}} \propto r^{-\eta}$, where $r$ is the distance to the AGN. Corresponding to this, the surface brightness profile of the undisturbed gas has the form, $\mathrm{SB} \propto \rho^{1-2 \eta}$, where $\rho$ is the projected distance from the AGN. The power law index $\eta$ was determined by fitting the SB profile in the region adjacent to the SW arm, outside the shock, giving $\eta=1.3$. Taking a cut perpendicular to the radius at a distance, $d$, from the AGN, gives the electron density profile $n_{\mathrm{e}, \mathrm{gas}} \propto\left(d^{2}+\varpi^{2}\right)^{-\eta / 2}$, where $\varpi$ is the cylindrical radius measured from the axis of the lobe. Using the distance, $d$, corresponding to $1.2^{\prime}$, this was the form adopt for the density profile of the undisturbed gas in the cylindrical flow model. The initial gas distribution was made hydrostatic by imposing a static gravitational field on the model. Shocks were initiated by depositing a large amount of energy on the symmetry axis and their evolution was followed using the cylindrically symmetric hydrodynamic code until the shock had expanded to the observed size. Surface brightness profiles for the model shocks were determined by projecting the axially symmetric models onto the sky, using the density and temperature dependent $0.3-1.8 \mathrm{keV}$ ACIS-I response. The temperature scale was determined by assuming a preshock temperature of $0.65 \mathrm{keV}$. The left panel of Fig. 8 shows the surface brightness profile of the SW shock, perpendicular to the radius, in the region shown in Fig. 7] together with model surface brightness profiles for Mach numbers of 1.42, 1.72 and 2.04 (computed assuming $\gamma=5 / 3$ in the gas). Based on its surface brightness profile in the vicinity of the shocks, the Mach 1.72 shock model provides the best fit.

The temperature jump expected for a Mach 1.72 shock is a factor of 1.74. For a preshock temperature of 0.65 $\mathrm{keV}$, that would make the postshock temperature $\simeq 1.13$ $\mathrm{keV}$. However, as observed, gas behind the cylindrical shock is projected onto the surrounding unshocked gas. The shocked gas also expands adiabatically behind the shock, reducing its average temperature. As a result, the observed temperature profile shows a considerably lower temperature jump than expected for a Mach 1.72 shock. Complicating matters further, fitted temperatures for multiphase gas are not readily determined from the emission measure distribution (Vikhlinin 2006). In view of this, projected emission measure distributions were determined for the Mach 1.72 model, assuming a preshock temperature of $0.65 \mathrm{keV}$. These were used to construct multiphase spectral models in XSPEC and the resulting models were fitted to a single temperature model to determine the observed temperature profile corresponding to the Mach 1.72 shock model. The results are plotted as stars together with the observed temperature profile in the right panel of Fig. 8. Within the errors, both the temperature and density profiles of this region are consistent with the Mach $\simeq 1.7$ shock model.

The calculations of the physical parameters derived from the model were performed for the SW bubble. The three cavities present similar temperature jumps and surface brightness profiles, so similar physical parameters are expected. From the hydrodynamic model, the age of the shock, $t \sim 2 \times 10^{6}$ yrs, is the time it takes to expand to its observed size. The age is only sensitive to the shock strength and the temperature of the unshocked gas. This age is slightly shorter than the ratio of the shock radius to the present shock speed, because in the model the shock strength decreases with time (i.e. the shock expanded faster when it was younger). The total energy which produced the shock was $10^{56}$ ergs, roughly equal to the enthalpy, $H=4 p V=7 \times 10^{55}$ ergs, calculated in the assumption that the bubble is predominantly relativistic $(\gamma=4 / 3)$. The average mechanical power required to produce the bubble equals to $P_{\text {mech }} \sim 1.6 \times 10^{42} \mathrm{erg} \mathrm{s}^{-1}$.

The age of the cavity is shorter than what is usually observed in the so-called 'ghost' cavities where no evident $1.4 \mathrm{GHz}$ radio emission is observed to fill them. However, the cavities in NGC 4636 are not really 'ghost' cavities and a weak radio luminosity is indeed expected from the mechanical power derived above. Bîrzan et al. (2008) found indeed a correlation (although with a large scatter) between the cavity (jet) power $P_{j e t}$ and the radio synchrotron power $L_{\text {radio }}$, of the form $P_{\text {jet }} \sim L_{\text {radio }}^{\beta}$. The cavities observed in NGC 4636 fall in the lower-left corner of the diagram in Figure 5 of Bîrzan et al. (2008), with a ratio $P_{\text {jet }} / L_{\text {radio }} \sim 10^{4}$, similar to a few objects in the plot presenting a radio luminosity four order of magnitudes brighter than NGC 4636. There is a large scatter in the correlation, that is not well understood. Given the associated jet powers, the low level of radio emission from $\mathrm{X}$-ray cavities in some of these objects is indeed surprising. As discussed in Bîrzan et al. (2008), the steep radio spectra in these systems cannot generally be explained by synchrotron aging or by adiabatic losses. Presumably some other loss mechanism is at work. This raises interesting questions on the nature of the plasma filling these cavities. As stated e.g. in Jetha et al. (2008), the nature of this material could be very hot, low-density plasma or could consist, at least in part, of a relativistic plasma containing relativistic electrons and magnetic field, similarly to that observed in the lobes of radio galaxies. In the case of NGC 4636, however, the complex X-ray morphology and the lack of low frequency radio maps (that will be presented in a forthcoming paper; Giacintucci et al. in prep.) does not allow at present to constrain the nature of the plasma filling the cavities (as it was done in e.g. Bîrzan et al. 2008; Jetha et al. 2008).

\subsection{The nuclear point source}

The X-ray morphology of NGC 4636 is characterized by the presence of a dense core having a radius of $\sim 1 \mathrm{kpc}$. A hard band image of the core $(2-7 \mathrm{keV}$ band) reveals the presence of several point sources. An X-ray point source is coincident with the radio nucleus (within $0.5^{\prime \prime}$ ) and may be X-ray emission from the supermassive black hole $(\mathrm{SMBH})$ at the center of the galaxy. We extracted a spectrum of this point source fitting a power-law model to it in XSPEC. The spectrum has $\sim 230$ counts (ACIS-S plus ACIS-I) in total and the best fit model gives a powerlaw index $\Gamma=2.5_{-0.5}^{+0.4}$ and a flux of $2.3 \pm 0.4 \times 10^{-15} \mathrm{erg}$ $\mathrm{cm}^{-2} \mathrm{~s}^{-1}$ and of $6.0 \pm 1.0 \times 10^{-15} \mathrm{erg} \mathrm{cm}^{-2} \mathrm{~s}^{-1}$ in the $2-10 \mathrm{keV}$ and in the $0.5-10 \mathrm{keV}$ bands, respectively. This corresponds to a total luminosity of $1.6 \pm 0.3 \times 10^{38} \mathrm{erg}$ $\mathrm{s}^{-1}$ in the $0.5-10 \mathrm{keV}$ band. This could be the current 
luminosity of the AGN at the center of NGC 4636. However, given the low luminosity, the X-ray emission could be also due to a low mass X-ray binary coincident with the radio nucleus. In the latter case, this value can be considered as a more stringent upper limit to the X-ray luminosity of the AGN at the center of the galaxy than the one set by Loewenstein et al. (2001).

To derive an estimate of the mass supply rate for accretion on the SMBH for NGC 4636, we can apply the Bondi (1952) theory of steady, spherical and adiabatic accretion. This requires $T$ and $n$ at "infinity", in practice near the accretion radius (that in the case of NGC 4636 is $r_{A}=G M_{B H} / c_{s}^{2} \sim 8 \mathrm{pc}$, where $c_{s}$ is the speed of sound). Unfortunately the spatial resolution does not allow us to measure the temperature and the density at such a small radius. However, we can estimate the accretion rate, using the temperature and the density profiles measured in in our radial analysis $\left(\S\left[\begin{array}{l}3.2 \\ )\end{array}\right)\right.$. The accretion rate is given by the formula

$$
\dot{M}_{\text {Bondi }}=8.4 \times 10^{21} M_{8}^{2} T_{0.5}^{-3 / 2} n_{1} g s^{-1},
$$

where $M_{8}$ is the SMBH mass in units of $10^{8} M_{\odot}$ (for NGC $4636 M_{B H} \simeq 7.9 \times 10^{7} M_{\odot}$, Merritt \& Ferrarese 2001), $T_{0.5}$ is the temperature in units of $0.5 \mathrm{keV}$ and $n_{1}$ is the density in units of $1 \mathrm{~cm}^{-3}$. Assuming that the density $n \propto r^{-1}$, from the thermal component normalization of the spectrum in the inner $5^{\prime \prime}(\sim 0.35 \mathrm{kpc})$, we obtain that the density at the accretion radius is $n \sim 5.5 \mathrm{~cm}^{-3}$. The temperature profile was instead fitted with a second order polynomial, giving a value at the accretion radius of $k T \sim 0.49 \mathrm{keV}$. Using this values of $n$ and $k T$, we find that $\dot{M}_{\text {Bondi }} \simeq 4.7 \times 10^{-4} M_{\odot} \mathrm{yr}^{-1}$. If at very small radius this gas joins an accretion disc with a standard radiative efficiency $\eta \sim 0.1$, as in brighter AGNs, it should produce a luminosity:

$$
L_{\text {acc }}=\eta \dot{M}_{\text {Bondi }} c^{2} \simeq 2.7 \times 10^{42} \mathrm{erg} \mathrm{s}^{-1},
$$

at least four orders of magnitude higher than the observed X-ray luminosity, pointing toward a highly inefficient accretion scenario.

\subsection{The bright core of $N G C 4636$}

The average density in the core is $\sim 0.1 \mathrm{~cm}^{-3}$ and the thermal pressure (computed as $2.2 n k T$, in the case that $\left.n=n_{p}\right)$ is $\sim 9.7 \times 10^{-11}$ dyn $\mathrm{cm}^{-2}$. Several substructures are visible as well as can be seen from Figure 11 where an $\mathrm{X}$-ray image of the central part of the galaxy is shown. This X-ray image (0.5-2 keV band) shows a cavity in the center of the core, although it is not clear, however, if we are dealing with a cavity or with a U-shaped enhancement in the surface brightness of the core. Interestingly, the position of this cavity is coincident with the inner radio jet detected at $1.4 \mathrm{GHz}$.

We performed a finer spatial and spectral analysis of the core region, considering the $\mathrm{X}$-ray emission west of the galaxy center $\left(315^{\circ}-405^{\circ}\right)$, where a sharper decline in surface brightness is detected. This sector was chosen also because the discontinuity matches the curvature of a wedge centered on the nucleus. The surface brightness profile in this sector is shown in Figure 12. In this profile, four regions with different behaviours can be identified easily. At the center a depression in the surface brightness (visible in Figure 11) is apparent. Outside the center $\left(2^{\prime \prime}<r<8^{\prime \prime}\right)$ we observe a flat region, followed by a sharp decline at $8^{\prime \prime}<r<18^{\prime \prime}$. At $r>18^{\prime \prime}$ the surface brightness flattens again then declines more gradually. We performed a spectral analysis in these four distinct regions to investigate variations in the temperature or in the abundance of the X-ray emitting gas. Because of the complexity of the emitting region and the number of counts detected we could not perform a meaningful deprojection, and we analyzed only the properties of the hot gas projected along the line of sight. A simple thermal model, plus a power-law to take into account the contribution of unresolved point sources, was considered (XSPEC model: wabs(apec+powerlaw)). The abundance was left free to vary in all the regions but the central $\left(r<2^{\prime \prime}\right)$, where it was fixed to 0.5 times solar because of the lower number of counts. Although a one temperature fit does not always account for the complexity of the spectra $\left(\chi_{\nu}^{2} \geq 1.3\right)$, it gives an indication of the general temperature and metal abundance trend. Temperature and abundance profiles for the sector are plotted in Figure 13. The temperature is constant at $2^{\prime \prime}<r<18^{\prime \prime}$ $(k T=0.56 \pm 0.01 \mathrm{keV})$ and it is slightly increasing right outside the core $(k T=0.60 \pm 0.01 \mathrm{keV})$. The sharp decline in surface brightness (a factor of at least 5 over a range of $10^{\prime \prime}$ ) is indicative of a drop in the density of a factor of at least 2. The drop in density is clearly not accompanied by a corresponding variation in the temperature, thus if the thermal pressure is the only acting force in the central region of NGC 4636, the bright core is not in pressure equilibrium with the ambient gas and cannot be a long lived structure. At least two possible scenarios can be invoked to explain the presence of the bright core. One scenario involves the presence of the radio lobes which created the cavities observed in NGC 4636, where the pressure induced by the relativistic plasma could restore the pressure equilibrium between the core and the ambient gas. In the other scenario the enhancement in brightness of the core region could be explained by an abundance gradient (as in e.g. NGC 507; Kraft et al. 2004). In Figure 13, a gradient in abundance between the flat region just outside the nucleus $\left(2^{\prime \prime}<r<8^{\prime \prime}\right)$, where $Z=1.0_{-0.1}^{+1.3} Z_{\odot}$, and the adjacent regions, where $Z \sim 0.6-0.7 Z_{\odot}$, is observed. This difference would not be enough to balance the pressure since the density is proportional to $Z^{-1 / 2}$, however the error bars are quite large and the fits demand for a more complex spectral model $\left(1.3 \leq \chi_{\nu}^{2} \leq 1.7\right)$. We performed a simple calculation to measure the diffusion timescale for Iron (and consequently for the other elements) to check whether the abundance gradient necessary to balance the pressure in the core could be long lived. For a thickness of the transition region (between the peak in surface brightness and the flattening) of $\sim 1 \mathrm{kpc}$, assuming Spitzer type collision cross-sections for Fe, a density of $0.1 \mathrm{~cm}^{-3}$, and a gas temperature of $k T \sim 0.5 \mathrm{keV}$, the timescale for $\mathrm{Fe}$ diffusion is a few $10^{13}$ yrs (i.e. several orders of magnitudes longer than a Hubble time). For the lower atomic weight elements the diffusion timescale would be shorter, going approximately as the atomic weight cubed (e.g. a factor of $\sim 40$ times shorter for $\mathrm{O}$ than $\mathrm{Fe}$ ). However, also considering lower atomic weight elements and all the 
possible uncertainties in the measure of the abundance (mostly due to the strong correlation between $k T, Z$ and flux in cool systems as NGC 4636), of the density and of the thickness of the transition region, the bright core could be very long lived if produced by an abundance gradient (and diffusion is covered by Coulomb collisions).

\section{CONCLUSIONS}

In this paper, we presented Chandra ACIS-I $(\sim 150 \mathrm{ks}$ integration time) and ACIS-S $(\sim 40 \mathrm{ks})$ observations of the X-ray luminous elliptical galaxy NGC 4636, located in the outskirts of the Virgo cluster. The main results can be summarized as follows:

- A soft band (0.5-2 keV) image shows the presence of a bright core in the center surrounded by an extended X-ray halo and two prominent quasisymmetric, $8 \mathrm{kpc}$ long, arm-like features, both of which are rims of ellipsoidal bubble-like structures (NE and SW bubbles). An additional bubble-like feature (E bubble) is detected just south of the NE bubble.

- We found that the temperature profiles across the bubble edges are sharp and characterized by the presence of temperature jumps of about $20-25 \%$. On the other hand, the metallicity across the rims is constant.

- Analyzing the core region of the galaxy we discovered the presence of a cavity around a weak twosided radio jet $\left(L \sim 1.4 \times 10^{38} \mathrm{erg} \mathrm{s}^{-1}\right)$. Several point sources are detected in the hard band (2-7 $\mathrm{keV}$ ) in the core region. One of the point sources is coincident with the central radio source; its low $\mathrm{X}$-ray luminosity of $1.6 \pm 0.3 \times 10^{38} \mathrm{erg} \mathrm{s}^{-1}$ may be consistent with that of a low mass X-ray binary coincident with a radio nucleus, however this luminosity may at least be considered as an upper limit to the present X-ray luminosity of the central AGN.

- We compared the observed temperature and surface brightness profiles across the bubble rims with numerical hydrodynamic shock models, demonstrating that a scenario where the bubbles were produced by shocks can explain the X-ray morphology. The shocks were probably driven by energy deposited off-center by jets originating from the central AGN. A further confirmation of this scenario is the presence of radio jets extending towards the bubbles and of a central weak X-ray and radio nucleus, most likely the currently observable signature of AGN activity, which was more intense in the past.

- For the SW bubble the shock has a Mach number of $\sim 1.7$, an age of $\sim 2 \times 10^{6}$ yrs, and a total energy required to produce the shock of $\sim 10^{56}$ ergs.

- A very bright dense $\left(n \sim 0.1 \mathrm{~cm}^{-3}\right)$ core of $\sim 1 \mathrm{kpc}$ $\left(15^{\prime \prime}\right)$ radius is observed in the galaxy center. The sharp decline in surface brightness (and consequently in density) in the core is not accompanied by a variation in temperature, not balancing the thermal pressure in the core with the ambient gas. This feature could be however long lived if the relativistic plasma injected beyond the core by the radio jets is balancing the thermal pressure or if the surface brightness enhancement is produced by an abundance gradient.

We thank J. Vrtilek and E. O'Sullivan for useful comments and discussion. We thank the anonymous referee for the useful comments and suggestions helpful to improve the presentation of the results in this paper.

\section{REFERENCES}

Anders, E., \& Grevesse, N. 1989, Geochim. Cosmochim. Acta, 53, 197

Arnaud, K. A. 1996, in Astronomical Society of the Pacific Conference Series, Vol. 101, Astronomical Data Analysis Software and Systems V, ed. G. H. Jacoby \& J. Barnes, 17-+

Awaki, H., Mushotzky, R., Tsuru, T., Fabian, A. C., Fukazawa, Y., Loewenstein, M., Makishima, K., Matsumoto, H., Matsushita, K., Mihara, T., Ohashi, T., Ricker, G. R., Serlemitsos, P. J., Tsusaka, Y., \& Yamazaki, T. 1994, PASJ, 46, L65

Bîrzan, L., McNamara, B. R., Nulsen, P. E. J., Carilli, C. L., \& Wise, M. W. 2008, ApJ, 686, 859

Bîrzan, L., Rafferty, D. A., McNamara, B. R., Wise, M. W., \& Nulsen, P. E. J. 2004, ApJ, 607, 800

Bondi, H. 1952, MNRAS, 112, 195

Brüggen, M., \& Kaiser, C. R. 2002, Nature, 418, 301

Buote, D. A. 2000, ApJ, 539, 172

Cavaliere, A., Lapi, A., \& Menci, N. 2002, ApJ, 581, L1

Churazov, E., Forman, W., Jones, C., \& Böhringer, H. 2000, A\&A, 356,788

Finoguenov, A., \& Jones, C. 2000, ApJ, 539, 603

Finoguenov, A., Ruszkowski, M., Jones, C., Brueggen, M., Vikhlinin, A., \& Mandel, E. 2008, ArXiv e-prints, 807

Forman, W., Jones, C., \& Tucker, W. 1985, ApJ, 293, 102

Forman, W., Nulsen, P., Heinz, S., Owen, F., Eilek, J., Vikhlinin, A., Markevitch, M., Kraft, R., Churazov, E., \& Jones, C. 2005, ApJ, 635, 894

Hoeft, M., \& Brüggen, M. 2004, ApJ, 617, 896
Jetha, N. N., Hardcastle, M. J., Babul, A., O'Sullivan, E., Ponman, T. J., Raychaudhury, S., \& Vrtilek, J. 2008, MNRAS, 384, 1344 Jones, C., Forman, W., Vikhlinin, A., Markevitch, M., David, L., Warmflash, A., Murray, S., \& Nulsen, P. E. J. 2002, ApJ, 567, L115

Kraft, R. P., Forman, W. R., Churazov, E., Laslo, N., Jones, C., Markevitch, M., Murray, S. S., \& Vikhlinin, A. 2004, ApJ, 601, 221

Loewenstein, M., Mushotzky, R. F., Angelini, L., Arnaud, K. A., \& Quataert, E. 2001, ApJ, 555, L21

Machacek, M., Nulsen, P. E. J., Jones, C., \& Forman, W. R. 2006, ApJ, 648, 947

Markevitch, M., Mazzotta, P., Vikhlinin, A., Burke, D., Butt, Y., David, L., Donnelly, H., Forman, W. R., Harris, D., Kim, D.-W., Virani, S., \& Vrtilek, J. 2003, ApJ, 586, L19

Matsushita, K., Makishima, K., Rokutanda, E., Yamasaki, N. Y., \& Ohashi, T. 1997, ApJ, 488, L125+

McNamara, B. R., \& Nulsen, P. E. J. 2007, ARA\&A, 45, 117

Merritt, D., \& Ferrarese, L. 2001, ApJ, 547, 140

Ohto, A., Kawano, N., \& Fukazawa, Y. 2003, PASJ, 55, 819

O'Sullivan, E., Ponman, T. J., \& Collins, R. S. 2003, MNRAS, 340, 1375

O’Sullivan, E., Vrtilek, J. M., \& Kempner, J. C. 2005, ApJ, 624, L77

Smith, R. K., Brickhouse, N. S., Liedahl, D. A., \& Raymond, J. C. 2001, ApJ, 556, L91 

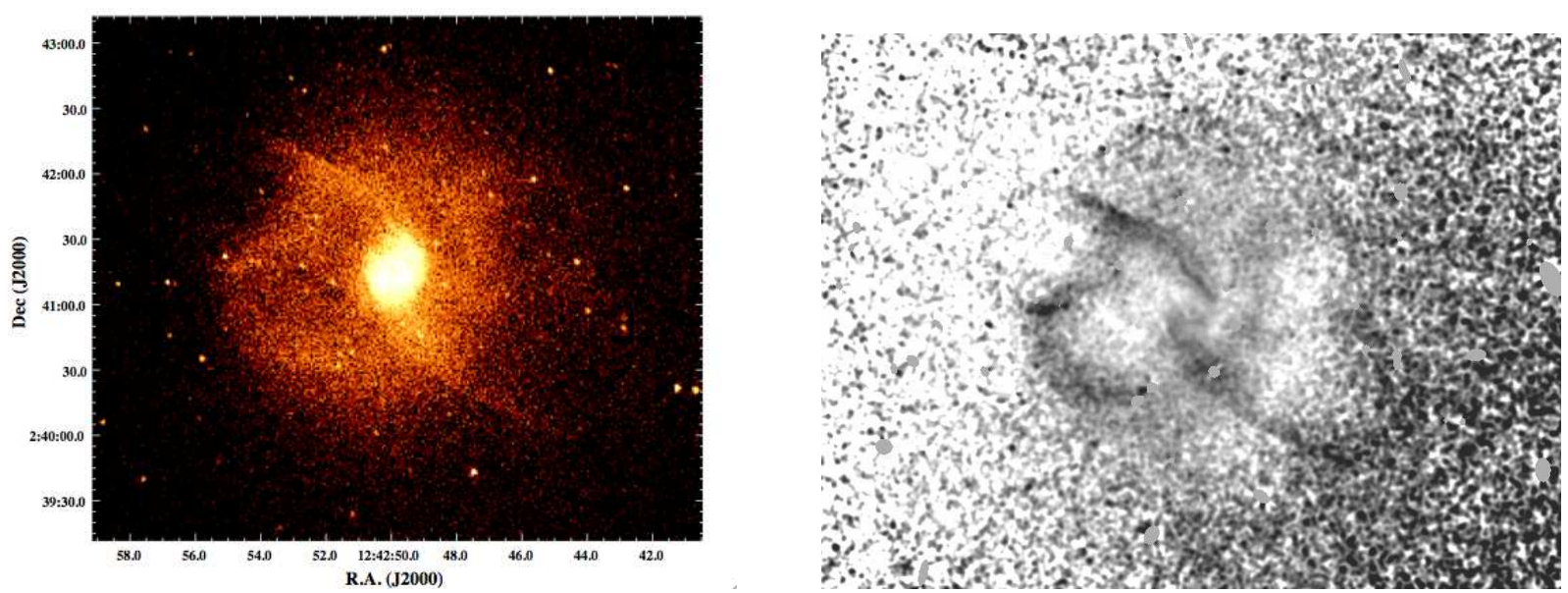

FIG. 1. - Left: Chandra ACIS-I+ACIS-S image of NGC 4636 in the $0.3-2 \mathrm{keV}$ band. The galaxy shows the presence of a bright central core $\left(r \sim 1 k p c \equiv 15^{\prime \prime}\right)$, surrounded by a lower surface brightness region extending as far as $\sim 6 \mathrm{kpc}\left(80^{\prime \prime}\right)$ from the center. Two pronounced quasi-symmetric ( $8 \mathrm{kpc}$ long) arm-like features define the rims of ellipsoidal bubbles. Also an additional bubble-like feature, whose northern rim is located $\sim 2 \mathrm{kpc}$ south of the north-eastern arm, is visible from the Chandra image. Right: Chandra ACIS-I+ACIS-S image after the subtraction of a $\beta$-model fitted to the general diffuse X-ray emission. The rims of the ellipsoidal bubbles are more clearly visible and another bubble almost symmetric to the bubble-like feature observed south of the north-eastern arm, could be present north-west of the nucleus.

Stark, A. A., Gammie, C. F., Wilson, R. W., Bally, J., Linke, R. A., Heiles, C., \& Hurwitz, M. 1992, ApJS, 79, 77

Tonry, J. L., Dressler, A., Blakeslee, J. P., Ajhar, E. A., Fletcher, A. B., Luppino, G. A., Metzger, M. R., \& Moore, C. B. 2001, ApJ, 546, 681

Trinchieri, G., Kim, D.-W., Fabbiano, G., \& Canizares, C. R. C. 1994, ApJ, 428, 555
Vikhlinin, A. 2006, ApJ, 640, 710

Vikhlinin, A., Markevitch, M., Murray, S. S., Jones, C., Forman, W., \& Van Speybroeck, L. 2005, ApJ, 628, 655 


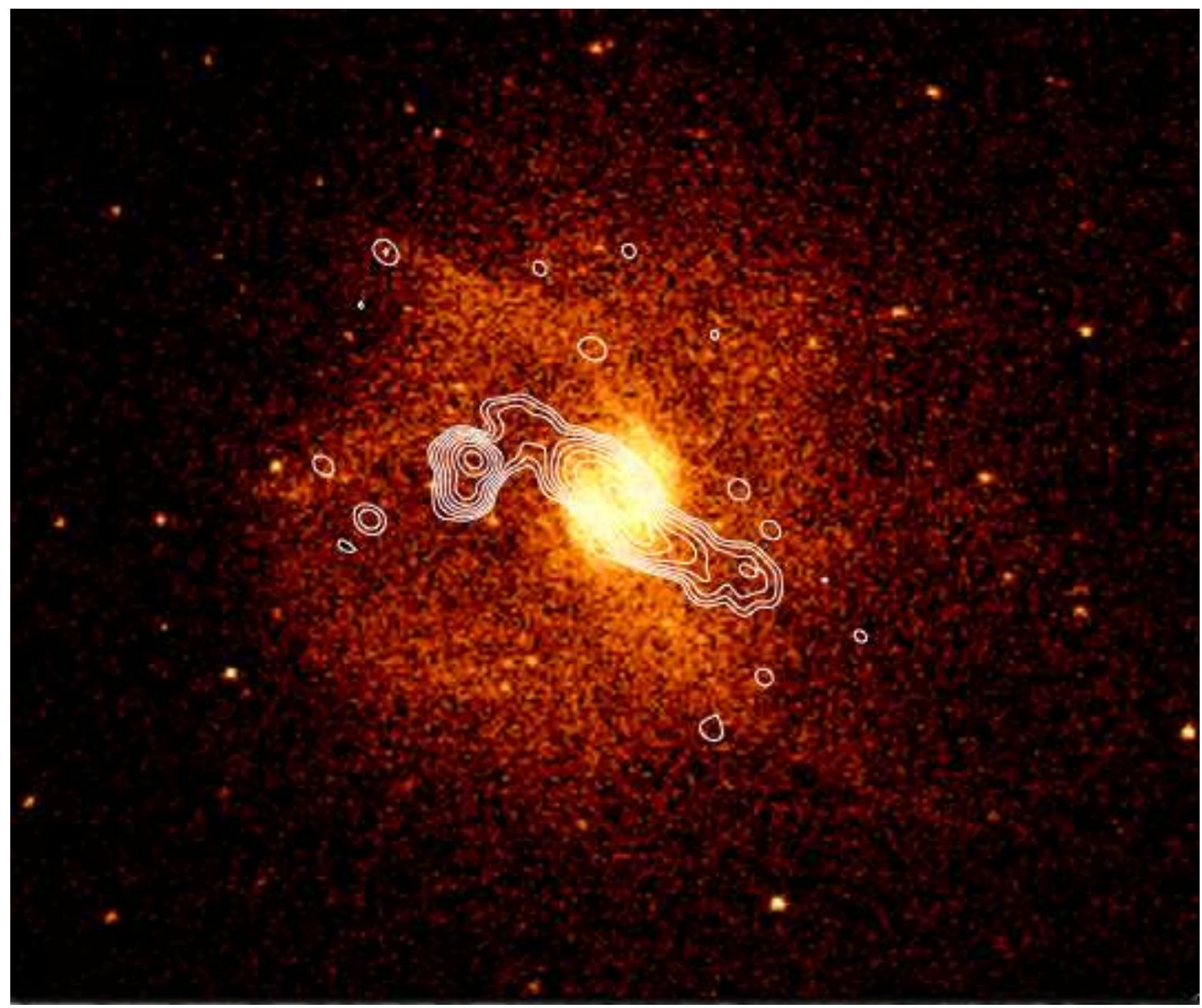

FIG. 2.- Chandra ACIS-I+ACIS-S image (0.5-3 keV) of NGC 4636 with the $610 \mathrm{MHz}$ GMRT radio contours superimposed (from Giacintucci et al. in prep.). The radio contours shows a correlation with the cavities, however they do not fill them completely, or break through the cavity boundaries. The resolution of the radio image is $5.8^{\prime \prime} \times 4.3^{\prime \prime}$, p.a. $48^{\circ}$, with the contours spaced by a factor 2 starting from $\pm 3 \sigma=0.15 \mathrm{mJy} /$ beam. An unrelated radio point-source is visible on the left of the nucleus, just below the NE cavity.
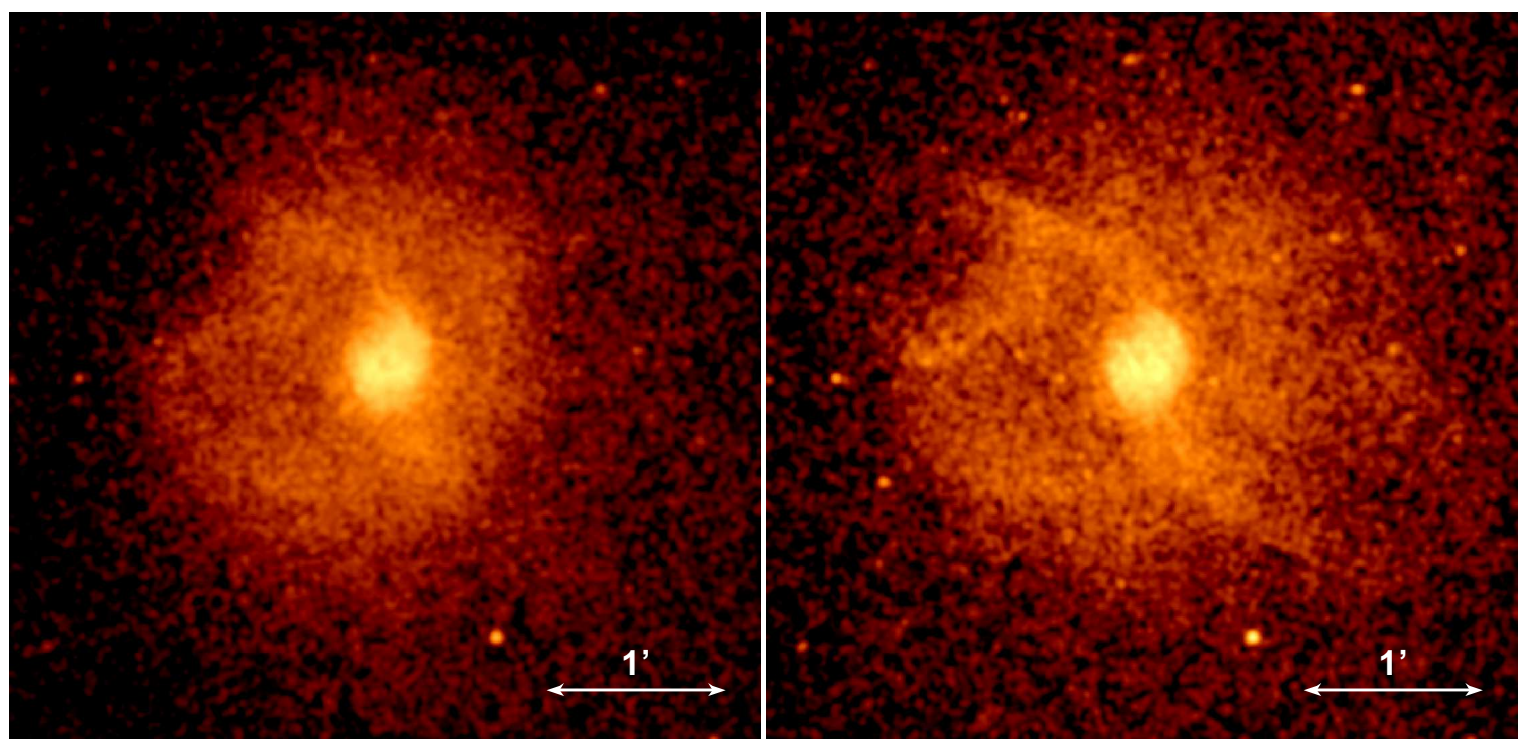

FIG. 3.- Left: $0.3-0.9 \mathrm{keV}$ Chandra ACIS-I+ACIS-S image of NGC 4636 smoothed with a 2" width gaussian; Right: 0.9-1.3 keV Chandra ACIS-I+ACIS-S image of NGC 4636 smoothed with a $2^{\prime \prime}$ width gaussian. The X-ray arm-like structures become prominent only in the higher energy band. In the figure $1^{\prime \prime}$ corresponds to $\sim 4.2 \mathrm{kpc}$. 


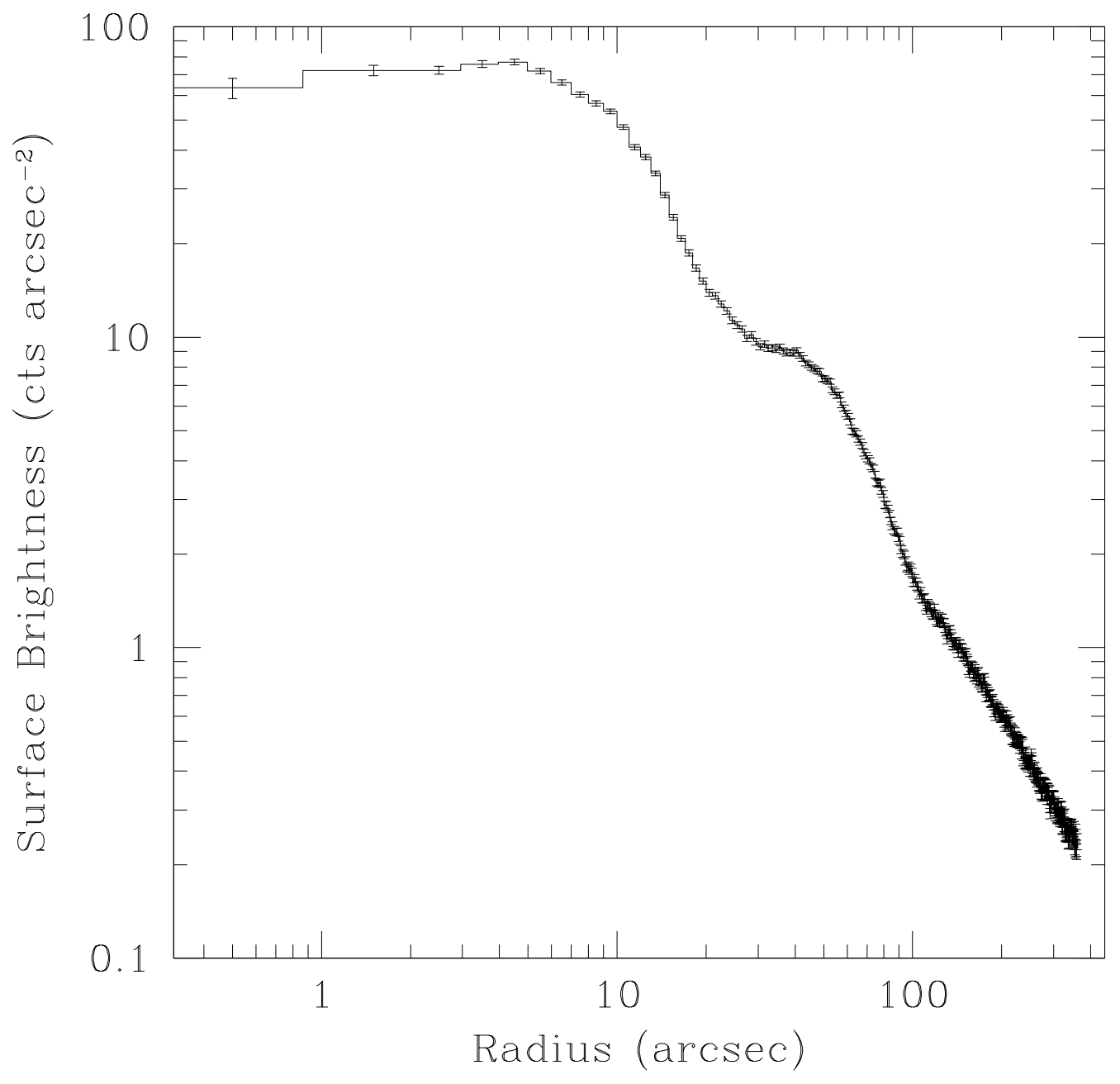

FIG. 4.- Chandra ACIS-I+ACIS-S surface brightness profile of NGC 4636 extracted in the 0.5-3 keV band. A central surface brightness peak is not present, being quite flat at the center. The profile declines monotonically at $5^{\prime \prime}<r<25^{\prime \prime}(0.35 \mathrm{kpc}<r<1.75 \mathrm{kpc})$, showing another 'plateau' at $25^{\prime \prime}<r<60^{\prime \prime}(1.75 \mathrm{kpc}<r<4.2 \mathrm{kpc})$. 

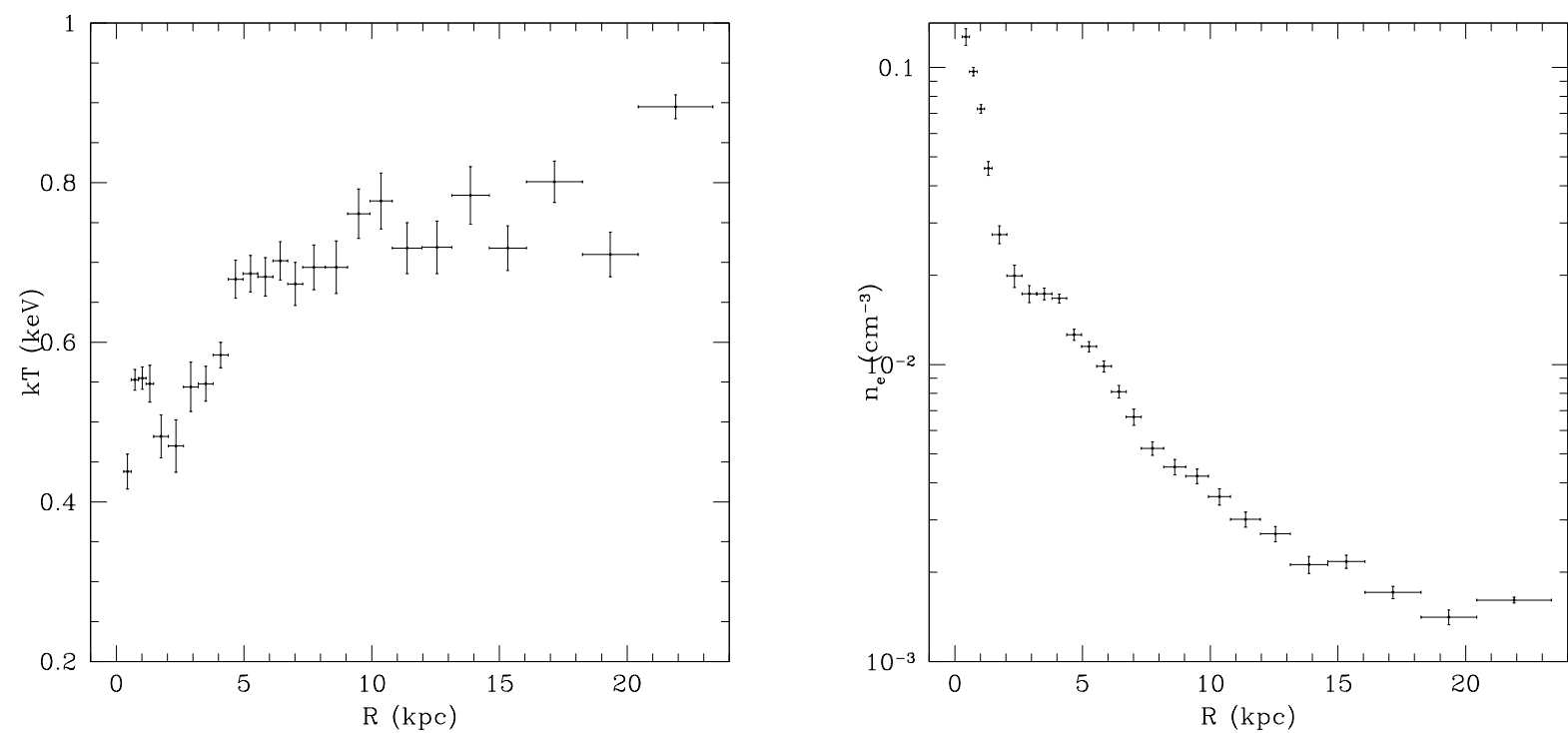

FIG. 5.- Left: Deprojected temperature profile for the X-ray gas observed in NGC 4636. A decline of the temperature is observed at the center, where $k T \sim 0.5 \mathrm{keV}$, while the temperature is increasing toward the outskirts of the galaxy $(k T \geqslant 0.8 \mathrm{keV})$. Right: Deprojected electron density profile for NGC 4636. The density shows the presence of a central peak and of a 'plateau' coincident with the flattening observed in the surface brightness profile, located at $2 \mathrm{kpc} \lesssim r \lesssim 4.5 \mathrm{kpc}$ from the center.

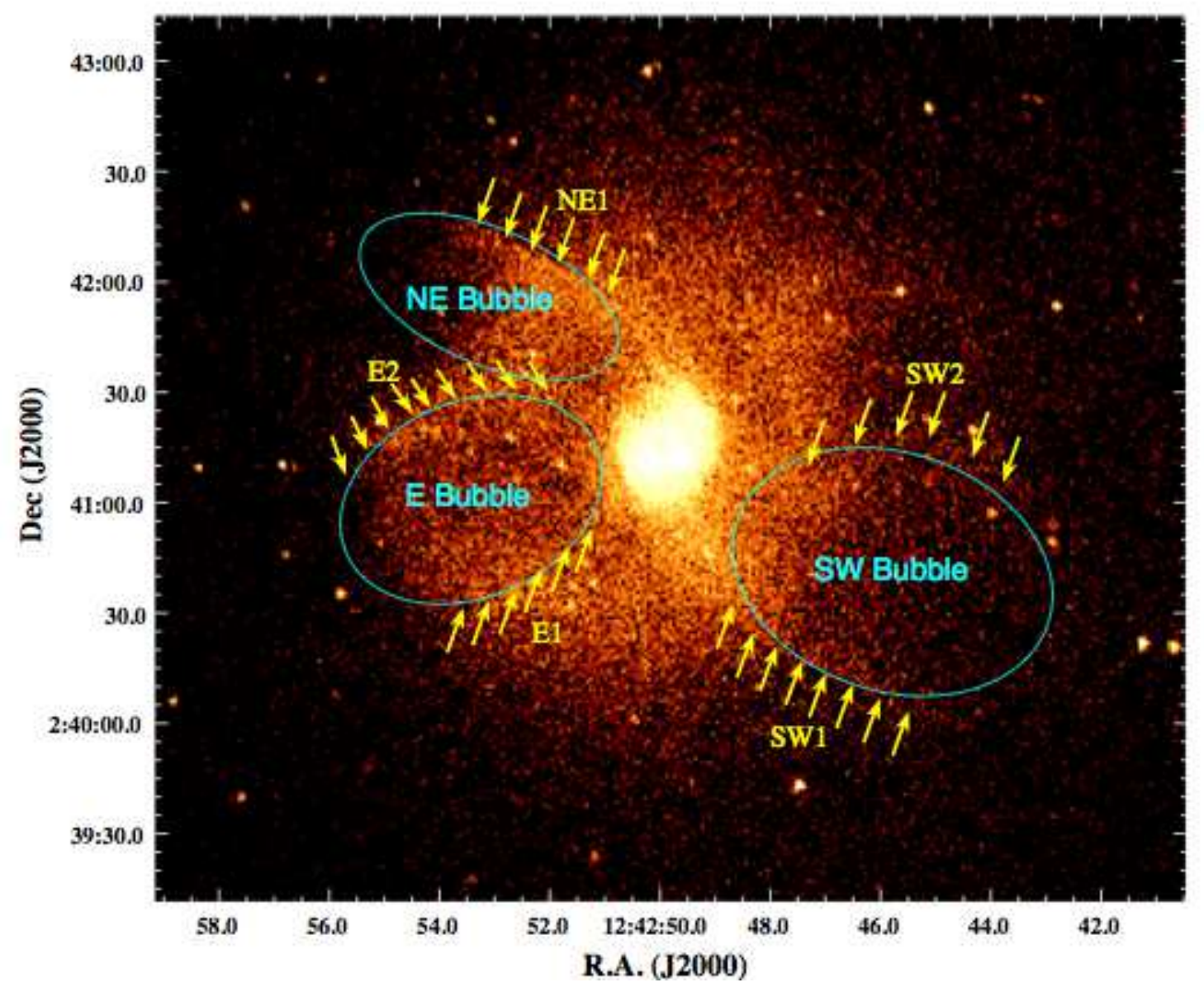

FIG. 6.- Chandra ACIS-I+ACIS-S image of NGC 4636 (0.5-3 keV band). The three bubble-like features detected are labelled and identified by cyan ellipses. The yellow arrows are pointing toward the detected rims of the bubbles. 


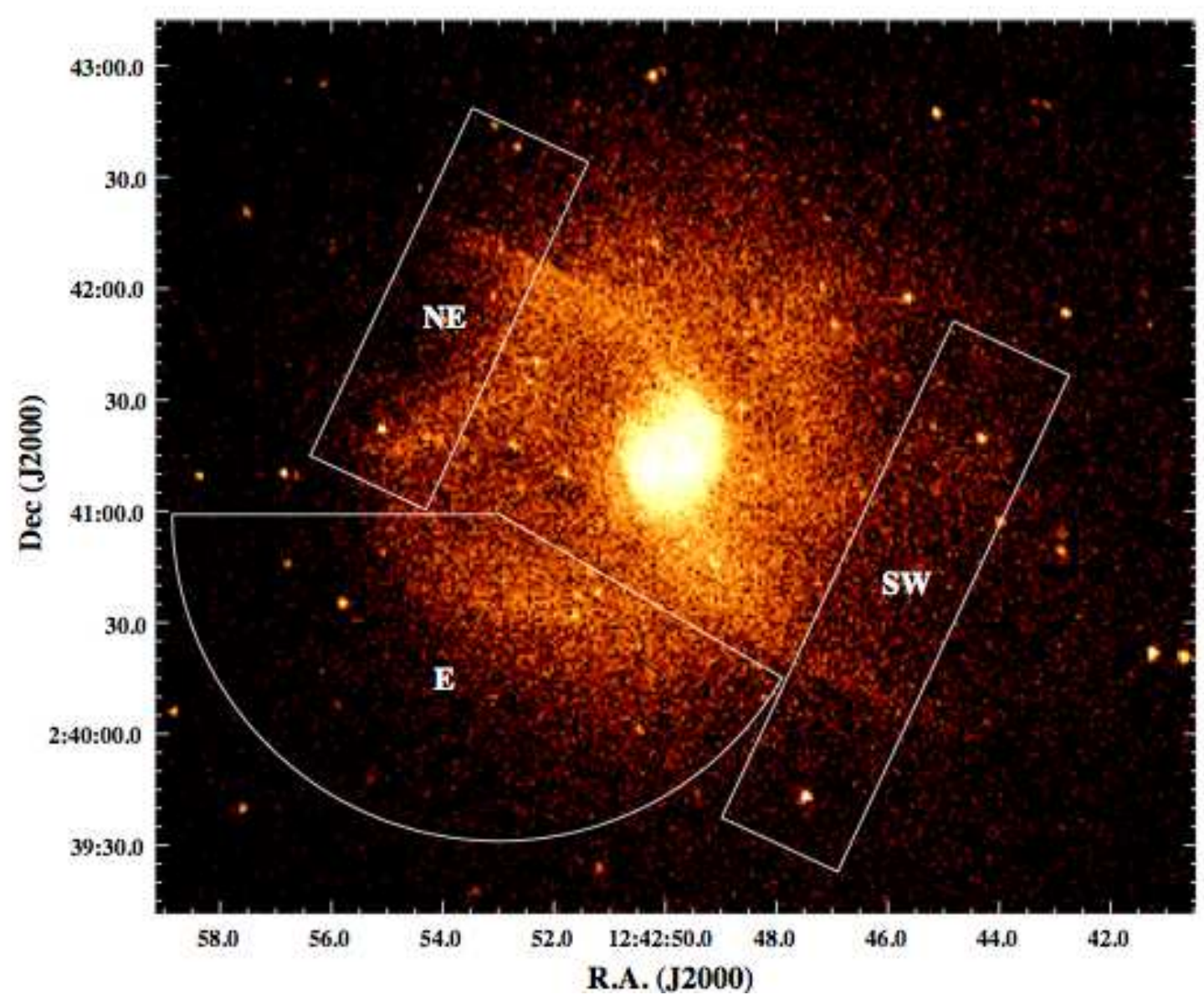

FIG. 7.- Chandra ACIS-I+ACIS-S image of NGC 4636 in the $0.5-3 \mathrm{keV}$ band. The regions used to extract the spectra for the analysis of the three bubble-like features are shown and labelled in white.
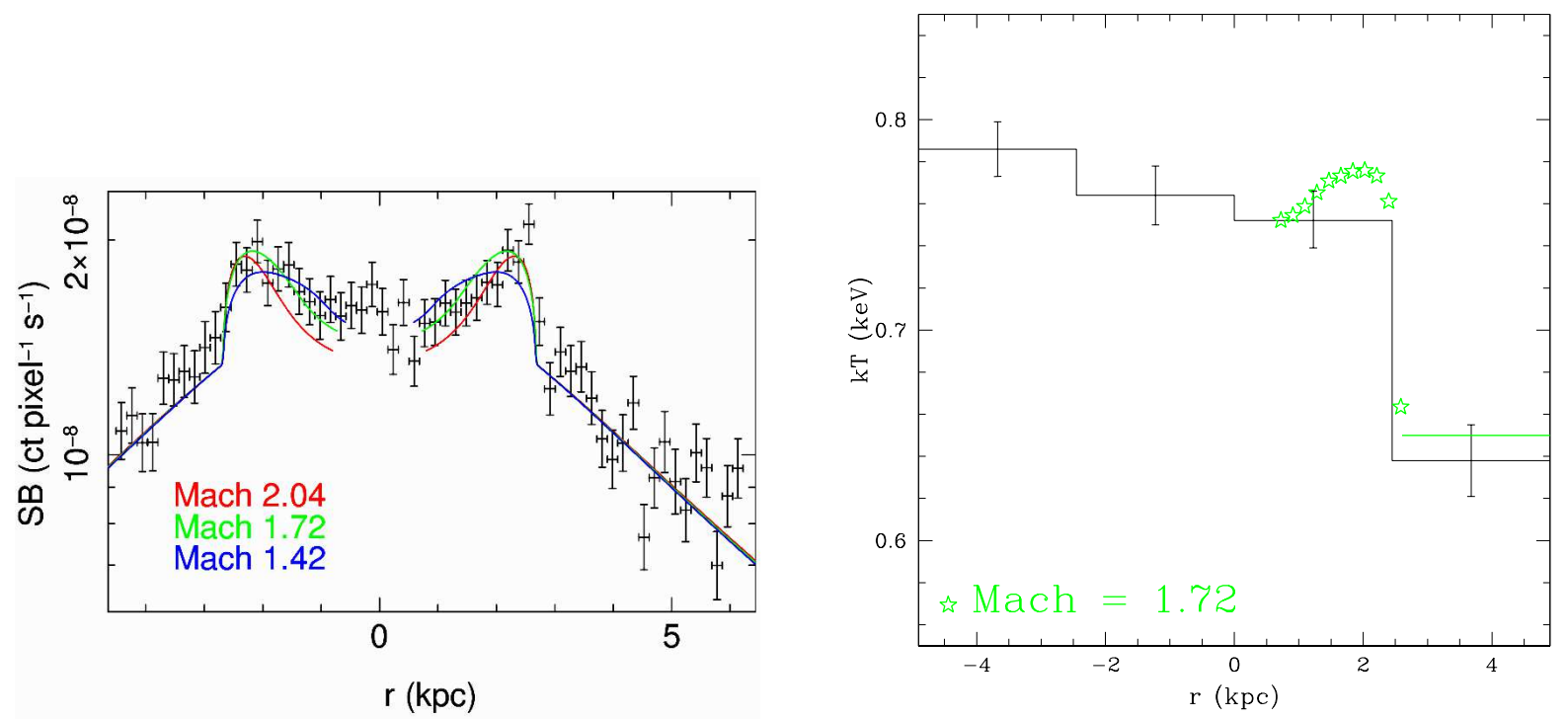

FIG. 8.- Left: Surface brightness profile perpendicular to the shock front for the SW bubble. The three colored solid lines represents the prediction from a numerical hydro-dynamical shock model at different Mach numbers for the shock. The best fit shock model to the observed data has $M=1.72$. Right: Temperature profile across the southern rim of the SW bubble. The temperature jump is consistent with the predictions of a shock model with $M=1.72$. The zero points in $r$ are coincident in the two panels and the values of $r$ increase toward the South. 

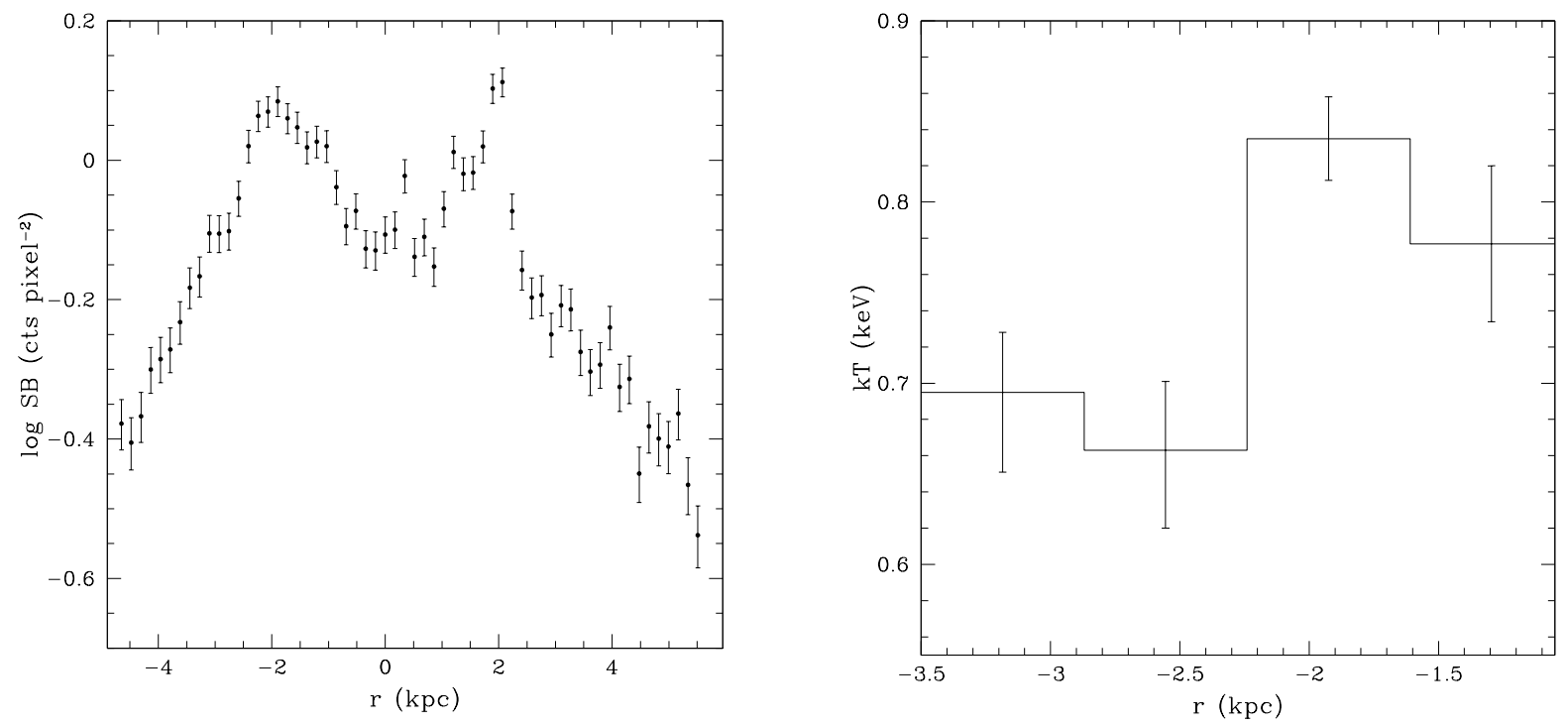

FIG. 9. - Left: Surface brightness profile perpendicular to the shock front for the NE bubble. Right: Temperature profile across the northern rim of the NE bubble. The shape of the surface brightness profile is very similar to the one observed in the SW bubble, moreover a temperature jump is observed in coincidence with the northern cavity rim (NE1). The zero points in $r$ are coincident in the two panels and the values of $r$ increase toward the South.
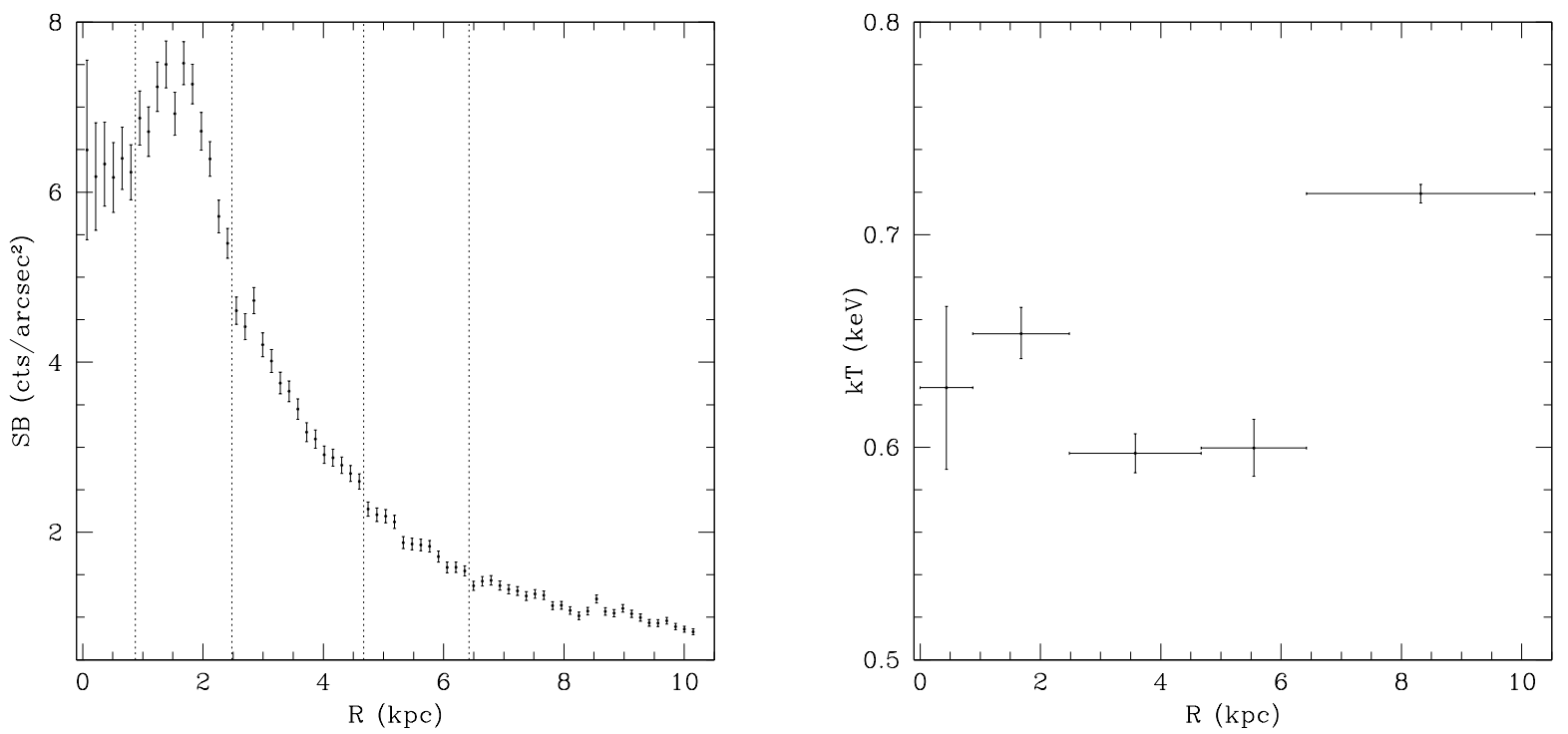

FIG. 10.- Left: Surface brightness profile across the southern rim for the East bubble. The dashed vertical lines delimitate the regions used for the spectral analysis. Right: Temperature profile across the southern rim of the E bubble. The shape of the surface brightness profile is analogous to the one observed in the other two bubbles and a temperature jump is observed in coincidence with the southern rim (E1) at $\sim 2.5 \mathrm{kpc}$ from the center of the bubble. 


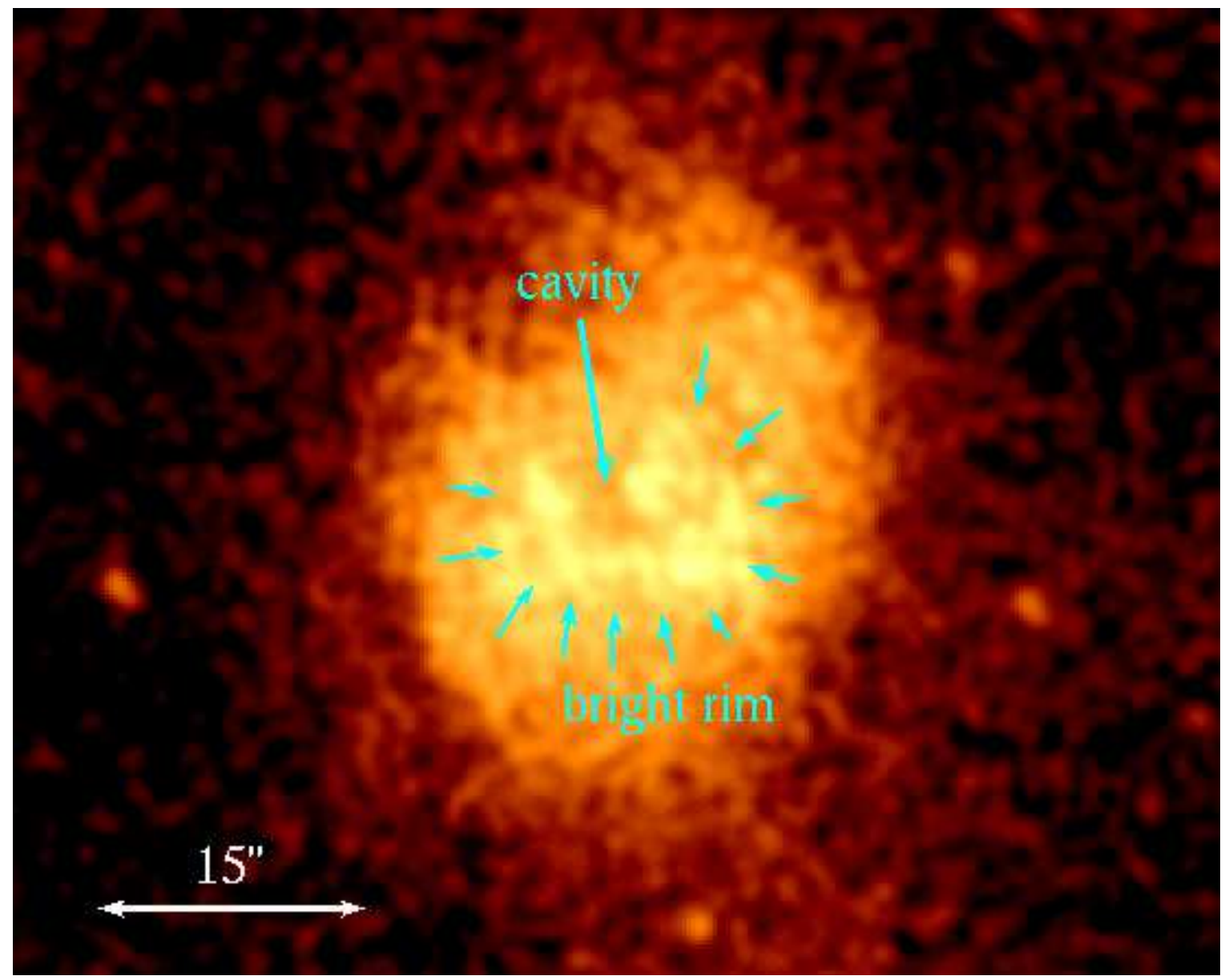

FIG. 11. - Chandra ACIS-I+ACIS-S image of the core of NGC 4636 (0.5-3 keV band). A cavity in the galaxy core is well visible and it is surrounded by a U-shaped brighter rim. 


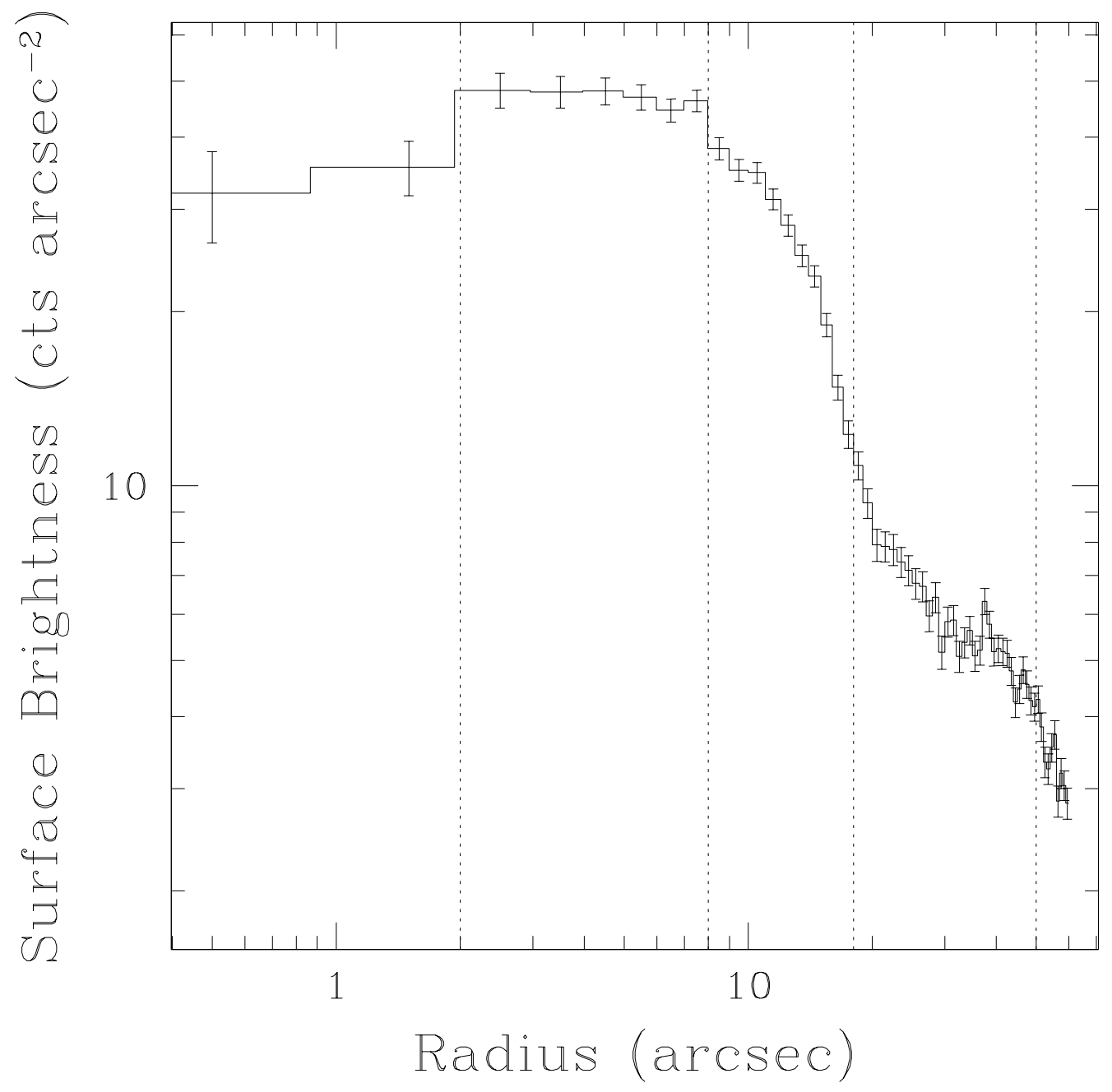

FIG. 12.- Surface brightness profile in a sector west of the nucleus $\left(315^{\circ}-405^{\circ}\right)$, extracted in the $0.5-3 \mathrm{keV}$ band. A depression in the center is well visible, followed by a flat region and then a sharp decline of the surface brightness. The dashed vertical lines delimitate the regions used for the spectral analysis. 

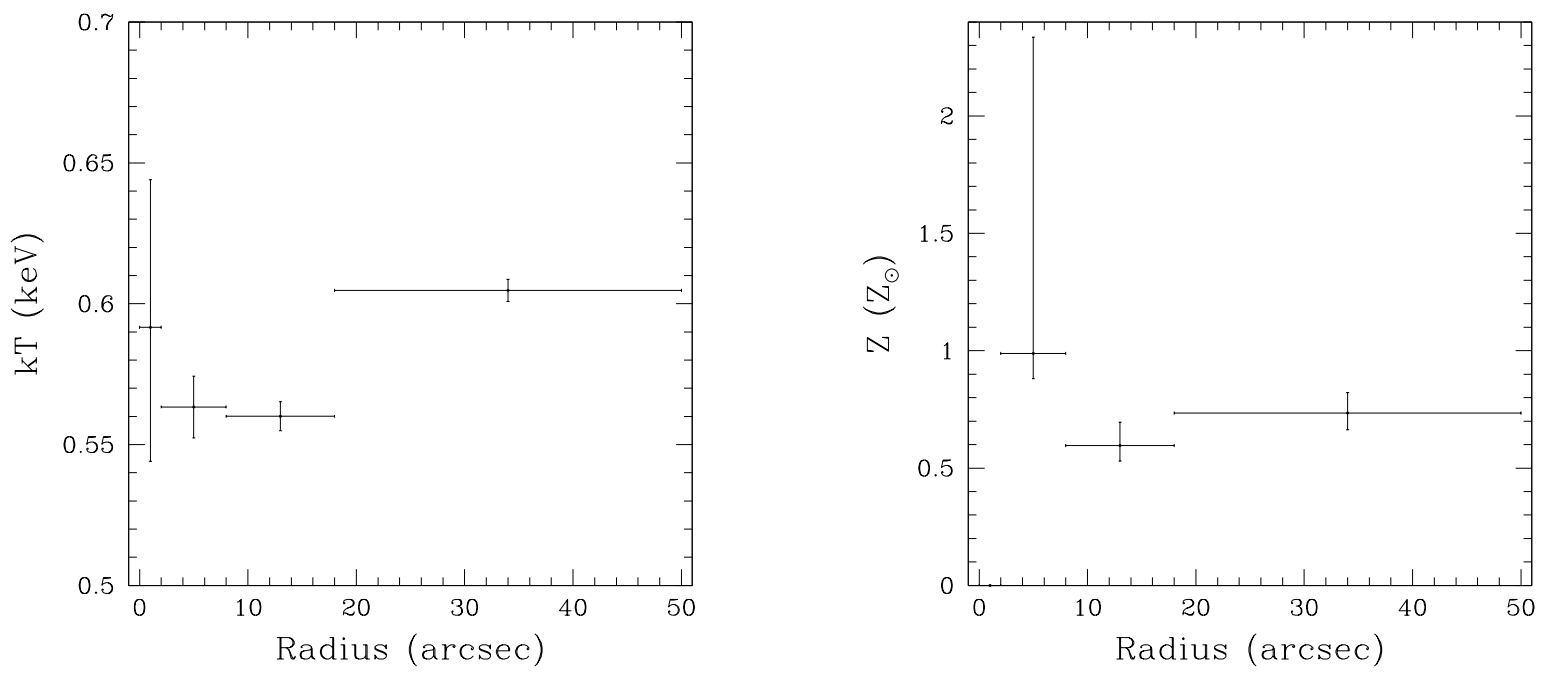

FIG. 13. - Left: Temperature profile in the $315^{\circ}-405^{\circ}$ sector. Right: Abundance profile in the $315^{\circ}-405^{\circ}$ sector. Both the temperature and the abundance profile are quite flat and do not present evidence of strong gradients with the radius. 